\title{
Las comunidades profesionales de aprendizaje, como estrategia de gestión de las escuelas que aprenden: El caso de una Institución Educativa estatal del Distrito de Ate.
}

\section{Professional learning communities, as a management strategy for schools that learn: The case of a state Educational Institution in the Ate District}

Guerra Cabrera, Gladys Liliana gladylili2104@gmail.com

https://orcid.org/0000-0002-4266-7634

Resumen: Las Comunidades Profesionales de Aprendizaje son una estrategia que favorece el desarrollo profesional docente y la mejora de los aprendizajes. La presente investigación, de enfoque cualitativo, tuvo como objetivo responder al problema ¿Cuáles son las percepciones del director y los coordinadores de grado sobre la gestión escolar desde la mirada de las comunidades profesionales de aprendizaje en una institución educativa del distrito de Ate?, y describir las dimensiones de las comunidades profesionales de aprendizaje que se presentan en la gestión escolar. El método es estudio de casos. Se aplicó la entrevista semiestructurada y el Focus Group a los informantes. Las dimensiones presentes en la Gestión escolar son: el Aprendizaje colectivo y su aplicación, el cual se percibe por la disposición de los docentes para trabajar en forma colegiada, estableciendo metas y compartiendo sus experiencias con sus pares para mejorar los aprendizajes, y la visión compartida que genera compromiso e identidad. Considerando los valores como: el respeto, la tolerancia y responsabilidad, el liderazgo del Director como factores importantes para el trabajo colaborativo.

Palabras clave: Comunidad Profesional de Aprendizaje (CPA), Organizaciones que aprenden (O.A.), Gestión escolar, trabajo colaborativo, Mejoramiento educativo

Abstract: Professional Learning Communities are a strategy that favors teacher professional development and improvement of learning. The present research with a qualitative approach aimed to respond to the problem What are the perceptions of the Director and the grade coordinators about school management from the perspective of the professional learning communities in an educational institution of the District of Ate ?, and describe the dimensions of professional learning communities that are presented in school management. The method is case study. The semi-structured Interview and the Focus Group were applied to the informants. The dimensions present in school management is collective learning and its application, they are perceived by the willingness of teachers to work in a collegiate way, they establish goals and share their experiences with their peers to improve learning. Also, the shared vision that generates commitment and identity. Considering values such as: respect, tolerance and

Experiencias de directivos de escuela: El rol del director: cómo lograr los aprendizajes de los estudiantes - Guerra, Gladys.

DOI: https://doi.org/10.18800/cige2020.010 
Congreso Internacional de Gestión Educativa, 06 y 07 de noviembre de 2020.

Pontificia Universidad Católica del Perú

responsibility, the Director's leadership as important factors for collaborative work, Educational Improvement.

Keywords: Professional Learning Communities (PLC), Learning Organizations, School management, collaborative work, educational Improvement.

\section{Introducción}

Una de las propuestas de mejora para la gestión escolar es la formación de comunidades profesionales de aprendizaje (CPA). Autores como Bolívar (2008, 2011), Bolivar (2012, 2013, 2017), Dufour (2004), Elboj \& Oliver (2003), Escudero (2009), Fielding (2001), Hord (1997, 2009), Krichesky \& Murillo (2011), Molina (2005), Murillo \& Krichesky (2012), señalan a las comunidades profesionales de aprendizaje como una estrategia orientada a mejorar las prácticas de enseñanza porque permiten repensar y rediseñar la institución educativa para la mejora escolar, centrada en los aprendizajes de los estudiantes y promoviendo una cultura de trabajo colaborativo.

Esta propuesta se basa en un aprendizaje dialógico y de interacción entre todos los miembros de la escuela (Elboj \& Oliver, 2003 y Hord, 1997). Se fundamenta en la pedagogía crítica, partiendo de la intención de investigar, analizar y actuar para promover una igualdad educativa y una mejora de sus prácticas pedagógicas, en beneficio de los aprendizajes de los estudiantes.

Las CPA, con la finalidad de seguir mejorando y aprendiendo, buscan responder a interrogantes como: ¿qué está enseñando la escuela?, ¿qué aprende la escuela a partir de sus propias experiencias como institución?, ¿qué medidas debería de adoptar para lograr sus objetivos? o como señalan Krichesky \& Murillo (2011), ¿está la escuela preparada para brindar a los profesores las condiciones necesarias para que éstos puedan ofrecer a sus estudiantes una enseñanza de calidad?

En ese sentido, la investigación realizada estuvo orientada a dar respuesta al problema ¿Cuáles son las percepciones del Director y los coordinadores de grado sobre la gestión escolar desde la mirada de las comunidades profesionales de aprendizaje en una Institución educativa del Distrito de Ate?

La investigación se sustenta en el marco teórico del Aprendizaje Organizacional (AO) "Organizational Learning", y las Organizaciones que aprenden (AO) "Learning Organization".

Asimismo, esta investigación se encuentra relacionada con las políticas emprendidas por el Ministerio de Educación sobre la gestión escolar desde el enfoque del liderazgo pedagógico centrado en los aprendizajes, así como en el marco del buen desempeño docente y el marco del buen desempeño directivo, los cuales buscan garantizar una educación de calidad, equidad y de inclusión en todas las instituciones educativas y fortalecer el trabajo colegiado.

Las investigaciones en torno a las Comunidades Profesionales de Aprendizaje (CPA) en el ámbito nacional, son escasas. Sin embargo, a nivel internacional, existen

Experiencias de directivos de escuela: El rol del director: cómo lograr los aprendizajes de los estudiantes - Guerra, Gladys.

DOI: https://doi.org/10.18800/cige2020.010 
Congreso Internacional de Gestión Educativa, 06 y 07 de noviembre de 2020.

Pontificia Universidad Católica del Perú

investigaciones realizadas en algunas escuelas norteamericanas, europeas y asiáticas sobre el impacto en las escuelas que implementan las CPA (PLC por sus siglas en inglés). Entre ellas se encuentran de Michele \& Santos (2016) en Singapur, Dogan, Tatik \& Yurtseven (2017) en Turquía, Voelkel \& Chrispeels (2017) en Estados Unidos, Kruse \& Johnson (2017) en Australia, Oh Song \& Choi (2017) en Corea, entre otros.

En ese sentido, la presente investigación abordó un campo aún no estudiado en el ámbito nacional, cuyo aporte se espera contribuya a implementar estrategias en la gestión escolar en nuestras escuelas para la mejora de los aprendizajes; a partir de la mirada de las Comunidades Profesionales de Aprendizaje, en el marco de las escuelas como organizaciones que aprenden.

La investigación corresponde al enfoque cualitativo y se realizó en base al método de estudio de casos. En el recojo de la información se emplearon técnicas de tipo cualitativo como el Focus Group, aplicado a los coordinadores de grado a través del instrumento del guión de Focus Group, y la entrevista semiestructurada con el instrumento guión de entrevista al Director de la institución educativa.

\section{Marco Conceptual}

\subsection{La Gestión escolar y las escuelas como organizaciones que aprenden.}

Si bien la función de la escuela es "enseñar a aprender", requiere también "saber aprender" para dar respuestas a las nuevas demandas sociales, a fin de evitar el fracaso y conseguir la mejora escolar a través de procesos autónomos, sin esperar reformas estructurales, impositivas y rígidas como señalan diversos autores (Bolívar, 2000; Duart, 1999; Murillo y Krichesky, 2014 y Santos, 2001).

En ese esfuerzo de cambios en las últimas décadas, el que ha tomado fuerza es la gestión escolar centrada en los procesos de enseñanza y aprendizaje, y la rendición de cuentas, como resultado de la gestión escolar.

Dos de los aportes teóricos de gran influencia frente a las deficientes estrategias de gestión para el cambio y la mejora escolar, son las teoría del "aprendizaje organizacional", Organizational Learning", (AO) y las "organizaciones que aprenden" Organizations Learning ${ }^{2}$ (OA). Estos planteamientos se presentan como "un nuevo paradigma del aprendizaje en el campo organizacional" (Rodríguez y Trujillo, 2007, p.100) logrando una gran notoriedad y relevancia con la publicación del texto "The fifth

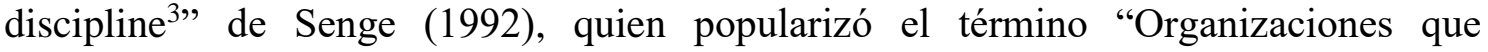
aprenden”, el cual despertó gran interés y fue adoptado en el ámbito educativo.

\footnotetext{
${ }^{1}$ Traducción libre "Aprendizaje organizacional" (A.O)

2 Traducción libre "Organizaciones que aprenden" (O.A.)

3 Traducción libre "La quinta disciplina"
}

Experiencias de directivos de escuela: El rol del director: cómo lograr los aprendizajes de los estudiantes - Guerra, Gladys.

DOI: https://doi.org/10.18800/cige2020.010 
Congreso Internacional de Gestión Educativa, 06 y 07 de noviembre de 2020.

Pontificia Universidad Católica del Perú

\subsubsection{Las organizaciones que aprenden en el ámbito educativo}

Para Leithwood \& Louis (1998), situar la imagen de la escuela como una "organización que aprende" parece ser una alternativa y respuesta prometedora a las continuas demandas de reconversión.

La concepción de las organizaciones como entidades "capaces de aprender", no sólo se limita al aprendizaje individual de los miembros que la componen, sino que promueve el aprendizaje colectivo (Senge, 1992). Estas organizaciones se sustentan en el fortalecimiento de las relaciones interpersonales que se dan en su interior a través del diálogo, el consenso, la consecución de metas y objetivos compartidos como aspiración colectiva e incluyen la evaluación de los procesos como una oportunidad para seguir creciendo basados en el trabajo colaborativo y una apertura al aprendizaje de manera ético- reflexiva.

La teoría de las organizaciones que aprenden (OA), se basa en el desarrollo de cinco disciplinas

El pensamiento sistémico, denominada la quinta disciplina, es un cuerpo de conocimientos que no pueden ser desarrollados sin una comprensión y mirada de procesos de interrelación con las demás disciplinas como un todo, un engranaje que funciona de manera interconectada. Está relacionado muy dependientemente de la visión compartida, porque no puede haber una visión sin un pensamiento sistémico que aglutine a los modelos mentales, al trabajo en equipo y al dominio personal para poder desarrollar todo su potencial.

Dominio personal esta disciplina es usada para referirse al crecimiento y el aprendizaje personal. Senge (1992) señala que "la gente con alto dominio personal expande continuamente su actitud para crear los resultados que buscan en la vida. De su búsqueda de aprendizaje continuo, surge el espíritu de la organización inteligente" (p. 181). Estas personas poseen un propósito especial que va más allá de su visión y metas. Asumen su realidad actual como un aliado, son conscientes de su ignorancia y sus zonas de crecimiento, tienen un alto sentido de responsabilidad, poseen iniciativas y aprenden con mayor rapidez.

Modelos mentales la disciplina de manejar los modelos mentales, empieza por volver la mirada hacia adentro: aprender a ver nuestras imágenes internas del mundo, para llevarlas a la superficie y someterlas a riguroso escrutinio (Senge, 2002). La ausencia de estos modelos hace que muchas buenas ideas no se concreten porque no se dan a conocer, o porque chocan con las imágenes internas que se encuentran muy arraigadas y son generalizaciones que influyen sobre nuestro modo de comprender el mundo y nuestra forma de actuar.

Visión compartida Senge (1992) afirma que la visión es una fuerza impresionante que es capaz de movilizar y generar un compromiso genuino de quienes se sienten identificados con sus propias visiones personales. Se crea un vínculo de identidad y una aspiración en común. La gente no aprende porque se lo ordenan, sino porque lo desea. La disposición de los individuos para con la visión no es de acatamiento sino

Experiencias de directivos de escuela: El rol del director: cómo lograr los aprendizajes de los estudiantes - Guerra, Gladys.

DOI: https://doi.org/10.18800/cige2020.010 
de "alistamiento" que significa incluirse, transformarse en parte de algo por propia elección, lo que hace que se sienta plenamente responsable de alcanzar la visión.

Aprendizaje en equipo esta disciplina implica ser capaz de dominar las prácticas habituales del diálogo y la discusión que son potencialidades de la organización. Nace de la idea de la ayuda mutua entre todos los miembros que la componen. Con este tipo de aprendizaje hay un propósito común, una visión compartida que permite aunar esfuerzos para desarrollar en los integrantes aptitudes extraordinarias para la acción coordinada. Bolívar (2001) afirma que cuando los equipos aprenden, se generan resultados extraordinarios porque sus integrantes crecen a la vez con una mayor rapidez y necesitan espacios para poder desarrollar la acción conjunta.

Estos aportes teóricos de las cinco disciplinas, han ido incorporándose como estrategias de innovación en la mejora de gestión escolar para hacer de las organizaciones entidades abiertas al cambio y fortalecer la gestión escolar y los aprendizajes.

\subsection{Las Comunidades Profesionales de Aprendizaje en el ámbito educativo.}

Bolívar (2008) y Molina (2005) señalan que las comunidades profesionales de aprendizaje (CPA) se pueden entender como una configuración práctica de las organizaciones que aprenden y como una nueva cultura de colaboración. Ello implica rediseñar la escuela, potenciar las capacidades internas de la organización y la reflexión crítica de la práctica docente. Supone desarrollar procesos de innovación y mayores niveles de poder en los miembros que la conforman para facilitar el fortalecimiento de las comunidades profesionales de aprendizaje, y la mejora del aprendizaje de los estudiantes en la gestión escolar (Krichesky y Murillo, 2011 y Bolívar, 2013).

\subsubsection{La formación de comunidades profesionales de aprendizaje como estrategia de la mejora escolar.}

La mejora escolar es entendida como un proceso permanente y de cambios en el cual la institución educativa optimiza el desarrollo integral de los estudiantes mediante el incremento de la calidad de sí misma y la de los docentes en forma colectiva, para lograr los aprendizajes de los estudiantes señalan (Fullan, 2002; Murillo y Krichesky 2014).

Una de las propuestas de mejora escolar es la formación de comunidades profesionales de aprendizaje (CPA). Bolívar (2008) y Molina (2005) manifiestan que la propuesta de la escuela como comunidad de aprendizaje representa un cambio fundamental en la comprensión y dinámica de la escuela, así como de la práctica profesional de los docentes, quienes están unidos por propósitos comunes y tienen un compromiso por aprender juntos de manera voluntaria.

No existe una definición universal y puede ser interpretada según diferentes contextos, Stoll, Bolam, McMahon, Wallace \& Thomas (2006) la definen como:

Experiencias de directivos de escuela: El rol del director: cómo lograr los aprendizajes de los estudiantes - Guerra, Gladys.

DOI: https://doi.org/10.18800/cige2020.010 
Congreso Internacional de Gestión Educativa, 06 y 07 de noviembre de 2020.

Pontificia Universidad Católica del Perú

"a group of people sharing and critically interrogating their practice in an ongoing, reflective, collaboritive, inclusive, learning oriented, growth promoting way, operating as a collective enterprise, ...blends process and anticiped outcome in defining a professional community of learners as one (P. 221). " 4

Por su parte, Leithwood, Seashore Louis, Anderson \& Wahlstrom (2004) sostienen que:

"The central idea of the model is the existence of a social architecture in school organizations that helps shape teachers' attitudes toward new pedagogies (Toole, 2001). Recent research using professional learning community as a variable has shown powerful associations with teacher practice (Bryk, Camburn and Louis, 1999; Louis, Marks and Kruse, 1999; Pounder, 1999; Scribner, Cockrell, Cockrell and Valentine, 1999; Toole, 2001). (p.66) $)^{5}$

Autores como Bolívar (2008; 2011), Bolívar (2012), Dufour (2004), Elboj \& Oliver (2003), Escudero (2009), Fielding (2001), Hord (1997; 2009), Krichesky y Murillo (2011), Molina (2005), Murillo y Krichesky (2014) definen a las comunidades profesionales de aprendizaje (CPA) como una estrategia orientada a mejorar las prácticas de enseñanza, pues permiten repensar y rediseñar la institución educativa para la mejora escolar, la cual pasa de ser una estructura burocrática a una organización que aprende en forma colaborativa.

\subsubsection{Dimensiones de las comunidades profesionales de aprendizaje.}

Tomando como referencia los trabajos teóricos y literatura al respecto, se pueden encontrar características comunes para configurar a las escuelas como comunidades profesionales de aprendizaje.

Existen cinco dimensiones claves que parecen estar entrelazadas y funcionan juntas. Stoll. et.al (2006) señala: Share values and vision, Collective responsibility, reflective professional inquiry, collaboration, Group, as well as individual, learning is promoted ${ }^{6}$.

\footnotetext{
${ }^{4}$ Traducción libre "un grupo de personas que comparten e interrogan críticamente sobre su práctica de una manera continua, reflexiva, colaboradora, inclusiva, orientada al aprendizaje, de promoción de la cultura, operando como una empresa colectiva, combina el proceso y resultado anticipado en la definición de una comunidad profesional de estudiantes"

5 Traducción libre "la idea central del modelo es la existencia de una arquitectura social en las organizaciones escolares que ayuda a moldear las actitudes de los docentes hacia las nuevas pedagogías (Toole, 2001). Investigaciones recientes que utilizan la comunidad de aprendizaje profesional como variable han demostrado poderosas asociaciones con la práctica docente (Bryk, Camburn y Louis, 1999; Louis, Marks y Kruse, 1999; Pounder, 1999; Scribner, Cockrell, Cockrell y Valentine, 1999; Toole, 2001).)

${ }^{6}$ Stoll, Bolam, Mcmahon, Wallace \& Thomas (2006) Compartir valores y visión, responsabilidad colectiva, investigación profesional reflexiva, colaboración, se promueve el aprendizaje grupal, así como individual.
}

Experiencias de directivos de escuela: El rol del director: cómo lograr los aprendizajes de los estudiantes - Guerra, Gladys.

DOI: https://doi.org/10.18800/cige2020.010 
Congreso Internacional de Gestión Educativa, 06 y 07 de noviembre de 2020.

Pontificia Universidad Católica del Perú

Para la investigación se tomaron en cuenta las dimensiones planteadas por Hord (1997), que fueron reafirmadas por Hipp \& Huffman (2003); Hipp \& Huffman, (2009) citados en Stoll et al.(2006).

\section{Liderazgo compartido y de apoyo.}

Mulford \& Sillins, (2003); Mulford, Sillins \& Leithwood (2004) citados en Krichesky y Murillo (2011) señalan que un factor importante en la CPA es la capacidad del liderazgo del director, el cual no está supeditado a las relaciones de poder, sino de crear las condiciones para una mayor implicación e involucramiento activo de los miembros que la integran a través de un liderazgo compartido que se promueva en todos los niveles, distribuyendo el poder en la escuela y brindando el apoyo necesario.

\section{Visión y valores compartidos.}

Bolívar (2008), Krichesky \& Murillo (2011), Stoll et al., (2006), afirman que la visión compartida debe ser real, clara, inspiradora, construida a partir del consenso de toda la comunidad y manifiesta un sentido de identidad. En tanto, los valores compartidos están dados por aquellas prácticas habituales que son incorporadas en las actuaciones de los miembros de la institución y son aceptadas socialmente.

Sin valores y metas compartidas en la organización, no hay sentido de comunidad (Andrews \& Lewis, 2007 citado en Pérez-Ruiz, 2014). La visión y valores compartidos forman la cultura de una institución y permanecen en el tiempo a través de los mitos, creencias y uso de símbolos de los miembros de la institución.

\section{Aprendizaje colectivo y su aplicación.}

En la CPA, de acuerdo, Bolívar (2013), Hord (1997) y Stoll et al., (2006) esta dimensión está referida al desarrollo de las relaciones conjuntas que llevan a cabo los docentes para mejorar los aprendizajes de los estudiantes y tienen una visión compartida. Elaboran juntos los procesos de planificación, resuelven problemas a partir de la reflexión de su práctica pedagógica y toman decisiones para mejorar las oportunidades de aprendizaje de forma colaborativa. Unas formas de identificar esas necesidades pueden darse a partir de los resultados de las evaluaciones de los estudiantes.

\section{Práctica profesional compartida.}

Según Stoll et al. (2006) esta dimensión está referida a cómo los docentes aprenden unos de otros. Es decir, todos son aprendices con sus colegas, el aprendizaje es en común y no en solitario. Los maestros desarrollan procesos de diálogo, pero también aprenden de sus pares a través de la observación de lo que su colega hace en el aula. Revisan su trabajo a la luz de los resultados apoyándose mutuamente tanto para la mejora individual como para la comunitaria (Bolívar, 2013).

Experiencias de directivos de escuela: El rol del director: cómo lograr los aprendizajes de los estudiantes - Guerra, Gladys.

DOI: https://doi.org/10.18800/cige2020.010 
Congreso Internacional de Gestión Educativa, 06 y 07 de noviembre de 2020.

Pontificia Universidad Católica del Perú

\section{Condiciones de apoyo: relaciones y estructura}

Los recursos y materiales disponibles como la infraestructura escolar, los espacios y tiempo deben estar orientados al fortalecimiento y desarrollo de la comunidad profesional de aprendizaje. Esto incluye favorecer el componente social-afectivo en las relaciones interpersonales, basado en el clima del respeto, la confianza, la motivación y el reconocimiento por lo individual y lo grupal.

Apertura, redes y alianzas, están referidas a fin de trascender los límites del centro escolar y en una actitud de apertura de los docentes a nuevas ideas, con la finalidad que se puede desarrollar alianzas con otras instituciones de modo que se apoyen de manera conjunta (Bolívar, 2008).

\subsubsection{Importancia de las Comunidades Profesional de Aprendizaje como estrategia de mejora en la gestión escolar.}

Tal como señala Bolam, McMahon, Stoll, Thomas, Wallace, Greenwood \& Smith (2005), la CPA es una herramienta valiosa para desarrollar la capacidad de la escuela y mejorar los aprendizajes, basado en las nuevas formas de trabajo colaborativo.

Stoll, Bolam, McMahon, Wallace \& Thomas (2006) quienes han hecho una revisión de la literatura en los últimos 25 años, señalan que existe un gran interés y arraigo por desarrollar este tipo de comunidades en las escuelas, por ser una estrategia que favorece el desarrollo profesional docente y la mejora de los aprendizaje.

Algunas de estas investigaciones se han llevado a cabo en las escuelas de los países de Norteamérica, Europa y Asia, entro otros.

Las investigaciones de Kruse \& Johnson (2017), Voelkel \& Chrispeels (2017), Oh Song \& Choi (2017) en las escuelas de Australia, California (EE.UU) y Corea, respectivamente, destacan la importancia y el impacto del liderazgo en las escuelas donde se desarrolla la CPA. Se afirma que un liderazgo informado permite trabajar respuestas bien informadas para resolver problemas del centro escolar y maximizar el aprendizaje. Por otro lado, la existencia de un liderazgo compartido y de apoyo que favorece las relaciones de colaboración entre docentes. La autonomía le otorga poder y autoridad a los docentes en las decisiones de manera participativa. Las características estructurales de soporte como el tiempo y los sistemas de comunicación son factores muy importantes.

Jones, Stall \& Yarbrough (2013) concluyen que se requiere mantener un equilibrio entre la teoría y la implementación de la CPA. Debido que a menudo se usan algunos términos con frecuencia pero las conceptualizaciones y pensamiento no se internalizan en los niveles intermedios. Por otro lado, Michele \& Santos (2016) en el estudio de algunas escuelas de Singapur, sobre el proceso de resolución colaborativa de problemas y el enfoque de aprendizaje, resultan ser desafiantes. Se brinda la oportunidad para examinar las ideas y compartir experiencias entre los colaboradores para atender el aprendizaje. Los docentes colaboran para organizar, planificar y evaluar las experiencias de aprendizaje de los alumnos, con el objetivo de mejorar esos aprendizajes.

Experiencias de directivos de escuela: El rol del director: cómo lograr los aprendizajes de los estudiantes - Guerra, Gladys.

DOI: https://doi.org/10.18800/cige2020.010 
Congreso Internacional de Gestión Educativa, 06 y 07 de noviembre de 2020.

Pontificia Universidad Católica del Perú

Dogan, Tatik \& Yurtseven (2017) evaluaron a las escuelas de Turquía con instrumentos ya validados y adaptados de Olivier, Hipp \& Huffman (2003) el PLCA-TR (cuestionario) cuyos resultados revelaron que existe una relación estrecha entre las dimensiones de las CPA, las cuales funcionan en forma sistémica. Además, señalan que este instrumento de evaluación puede servir para realizar diagnósticos para la toma de decisiones y cómo se encuentra cada una de ellas en la escuela.

Estas investigaciones demuestran el impacto de la CPA en su implementación pero, a su vez, evidencian las necesidades a mejorar. Asimismo, los estudios han demostrado que existe una relación en los resultados de rendimiento académico de los estudiantes, asociados a las escuelas donde se desarrollan las comunidades profesionales de aprendizaje (CPA).

\section{Diseño Metodológico}

La investigación se centró en dar respuesta al problema ¿Cuáles son las percepciones del Director y los coordinadores de grado sobre la gestión escolar desde la mirada de las comunidades profesionales de aprendizaje en una Institución educativa del Distrito de Ate?

La investigación corresponde al enfoque cualitativo, porque estudia la realidad, tal y como sucede de acuerdo con las personas implicadas (Flick, 2004; Pérez, 1998; Taylor \& Bogdan, 1996; Schwandt, 1994, citado en Latorre, A., Del Rincón, D. y Arnal, J. 1996). El método fue el estudio de casos, que tuvo como propósito "la descripción y análisis detallado de unidades sociales o entidades educativas únicas" (Yin, 1989 citado en Latorre et al., 1996.p.233), Stake, 1998.

En este caso una institución educativa de gestión estatal que pertenece al nivel primaria, ubicado en el Distrito de Ate. Cuenta con alta demanda de estudiantes y prestigio en la zona. Ha sido reconocida por los niveles de logro de aprendizajes en las evaluaciones censales del Ministerio de Educación (ECE). El objetivo fue analizar cómo se percibe la gestión escolar desde la mirada de las comunidades profesionales de aprendizaje e identificar qué dimensiones se presentan en la gestión escolar de la institución educativa desde las percepciones del director y los coordinadores de grado.

La categoría: Dimensiones de la CPA y las sub categorías son: Liderazgo compartido y de apoyo; visión y valores compartidos; aprendizaje colectivo y su aplicación, práctica profesional compartida y las condiciones de apoyo: relaciones y estructuras.

Se utilizaron técnicas de inmersión interactivas de tipo cualitativa como: la Entrevista semiestructurada al Director de la institución educativa y el Focus group a los coordinadores de grado. Los instrumentos fueron validados por juicio de expertos con docentes de experiencia de la PUCP y se realizó una prueba piloto previa.

Para el análisis y discusión de los resultados se procedió a transcribir la información recogida a través de los instrumentos: Guión de Focus Group y Guía de Entrevista semi estructurada registradas en audios, luego se codificó de acuerdo a las Categorías y sub categorías de la CPA, así como la información emergente contrastándolas a partir de las percepciones de los informantes, respetando el principio ético de la investigación.

Experiencias de directivos de escuela: El rol del director: cómo lograr los aprendizajes de los estudiantes - Guerra, Gladys.

DOI: https://doi.org/10.18800/cige2020.010 
Congreso Internacional de Gestión Educativa, 06 y 07 de noviembre de 2020.

Pontificia Universidad Católica del Perú

\section{Conclusiones}

De acuerdo a los objetivos de la investigación se concluye que las dimensiones de la CPA que están presentes en la gestión escolar de la I.E desde la percepción de los informantes son:

- El aprendizaje colectivo y su aplicación, se evidencia en la disposición que tienen los docentes para el trabajo en forma colegiada y de manera voluntaria. En este espacio analizan y reflexionan sobre su práctica pedagógica, los resultados de aprendizaje de los estudiantes, comparten experiencias con sus pares para mejorar sus estrategias de enseñanza y asegurar los resultados de aprendizaje de los estudiantes. Se apoyan y aprenden de manera conjunta evitando el individualismo, siendo un gran dinamizador el liderazgo pedagógico del Director.

- La visión y valores compartidos, la visión construida de manera participativa y por consenso, genera compromiso de los miembros de la comunidad y de los que se incorporan a la institución educativa. Está centrada en la mejora de los aprendizajes y consignados en los instrumentos de gestión. Los valores como la responsabilidad, el respeto y la tolerancia están arraigados y son necesarios para desarrollar el trabajo colaborativo y un buen clima escolar. Estos comportamientos se muestra en el actuar de los docentes que laboran en ella..

- El liderazgo compartido y de apoyo se delega tareas y responsabilidades entre los docentes a través de comisiones de trabajo, que les da poder y autoridad en la institución educativa dándoles autonomía en el desarrollo de sus labores.

Las demás dimensiones se encuentran en proceso debido a la situación de dependencia y de los escasos recursos económicos que son limitaciones de las instituciones educativas públicas.

\section{Recomendaciones}

- Desde el aspecto metodológico, seguir investigando en las instituciones educativas que estén implementando la CPA, usando las herramientas ya elaboradas (cuestionarios) para recoger información de otros miembros de la comunidad educativa como: padres de familia, personal administrativo, estudiantes, aliados estratégicos y otros que dan soporte a la gestión escolar.

- Promover la autonomía de la gestión escolar para rediseñar los horarios de trabajo, a fin de promover espacios para la reflexión de la práctica pedagógica en conjunto y sean remuneradas, tal como sucede en la educación secundaria, a fin de fortalecer el trabajo colaborativo para la mejora de los aprendizajes.

- Interiorizar en los miembros de la comunidad educativa donde se implementan la CPA los aspectos conceptuales de las dimensiones que se encuentran presentes en la gestión escolar dadas por el Ministerio de educación de manera fragmentada.

Experiencias de directivos de escuela: El rol del director: cómo lograr los aprendizajes de los estudiantes - Guerra, Gladys.

DOI: https://doi.org/10.18800/cige2020.010 
Congreso Internacional de Gestión Educativa, 06 y 07 de noviembre de 2020.

Pontificia Universidad Católica del Perú

- Promover e implementar la formación de comunidades profesionales de aprendizaje como estrategia de gestión escolar para la mejora de los aprendizajes y el desarrollo profesional de los docentes, brindando el apoyo y soporte que requieren para su consolidación en el tiempo, reconociendo a la escuela como un lugar para enseñar, pero también para aprender.

Agradecer las facilidades brindadas por el Director y coordinadores de grado de la institución educativa para el desarrollo de esta investigación, así como de los docentes asesores de la Escuela de Post grado de la PUCP, por sus aportes para la investigación y a mi asesor de tesis Dr. Alex Oswaldo Sánchez Huarcaya.

\section{Referencias bibliográficas}

Bolam, R., McMahon, A., Stoll, L., Thomas, S., Wallace, M., Greenwood, A., Smith, M. (2005). Creating and sustaining effective professional learning communities. Research Report 637. London, UK: Universities of Bristol. Recuperado de: https://dera.ioe.ac. uk/5622/1/RR637.pdf

Bolívar, A. (2000). Los centros educativos como organizaciones que aprenden: Promesa $\quad y$ realidades. Madrid: Editorial La Muralla.

Bolívar, A. (2001). Los centros educativos como organizaciones que aprenden. Una mirada crítica. Revista Contexto Educativo, (18), 1-11. Recuperado de: https:// www.researchgate.net/profile/Antonio_Bolivar/publication/4813873

7_Los_centros_educativos_como_organizaciones_que_aprenden/links/55e $57 \mathrm{~d} 3508 \mathrm{aebdc}$ 0f589da6f/Los-centros-educativos-como-organizaciones-que-aprenden.pdf

Bolívar, A. (2008). Otra alternativa de innovación, las comunidades profesionales de aprendizaje. Researchgate. XIII Congreso de UECOE "Educar: Innovar para la transformación social" Gijón (22-24 octubre 2008). Recuperado de: https://researchga gate.net/profile/Antonio_Bolivar/publication/286060811_Otra_alternativa_de_innovacion _las_comunidades_profesionales_de_aprendizaje/links/5665bf5008ae192bbf9260da/Otra -alternativa-de-innovacion-las-comunidades-profesionales-de-aprendizaje.pdf

Bolívar, A. (2011). Aprender a liderar líderes. Competencias para un liderazgo directivo que promueva el liderazgo docente. EDUCAR, 47 (2), 253-275. Recuperado de: http://www.redalyc.org/html/3421/342130837004/

Bolívar, R. M. (2012). La cultura de aprendizaje de las organizaciones educativas. Instrumentos de diagnóstico y evaluación, REICE. Revista Iberoamérica sobre Calidad, Eficacia y

Experiencias de directivos de escuela: El rol del director: cómo lograr los aprendizajes de los estudiantes - Guerra, Gladys.

DOI: https://doi.org/10.18800/cige2020.010 
Congreso Internacional de Gestión Educativa, 06 y 07 de noviembre de 2020.

Pontificia Universidad Católica del Perú

Cambio en Educación, 10 (1), 143-162. Recuperado de: http://www.redalyc.org/pdf/551/55123361010.pdf

Bolívar, M. (2013). Comunidades profesionales de aprendizaje. Instrumentos de diagnóstico y evaluación. Revista Iberoamericana de Educación, 62(1), 1-12. Recuperado de: https://www.redalyc.org/pdf

Bolívar, M. (2017). Los centros escolares como comunidades de aprendizaje. (Tesis doctoral). Universidad de Granada. España. Recuperado de: https://hera.ugr.es/tesisugr/2647 9436.pdf

Dogan, S., Tatık, S., y Yurtseven, N. (2017). Professional Learning Communities Assessment: Adaptation, Internal Validity, and Multidimensional Model Testing in Turkish Context. Educational \& Sciencies: Theory \&Practice, 17(4), 1203-1229.doi:10.12738/estp.

2017.4.0479

Duart, J.M. (1999). La organización ética de la escuela y la transmisión de Valores. Barcelona. España: Paidós

DuFour, R. (2004). What is a "professional learning community? Educational Leadership. 61 (8), 6-11. Recuperado de: http://citeseerx.ist.psu.edu/viewdoc/download?doi=10. 1.620.3269\&rep=rep1\&type $=$ pdf

Elboj, C. \& Oliver, E. (2003). Las comunidades de aprendizaje: Un modelo de educación dialógica en la sociedad del conocimiento. Revista Interuniversitaria de formación del profesorado, 17 (3), 2003 91-103. Recuperado de: http://www.redalyc.org/html/274/

27417306/

Escudero, J. (2009). Comunidades de aprendizaje. Formación de profesorado y mejora de la educación. Teacher learning communities. Teacher's education and school improvement. Ágora para la E.F. y el deporte. $\mathrm{N}^{\circ}$ 10, 2009, 7-31. Recuperado de: http://uvadoc.uva.

es/bitstream/10324/23683/1/AEFD-2009-10-comunidades-docentes.pdf

Fielding, M. (2001). Learning organization or learning community? A critique of Senge. Reason and practice, 1 (2), 17-29. Recuperado de: https://www.researchgate.net/ profile/Michael_Fielding/publication/271313371_Learning_Organisation_or_Learning_C ommunity/links/54e20cc40cf2966637941185.pdf

Experiencias de directivos de escuela: El rol del director: cómo lograr los aprendizajes de los estudiantes - Guerra, Gladys.

DOI: https://doi.org/10.18800/cige2020.010 
Congreso Internacional de Gestión Educativa, 06 y 07 de noviembre de 2020.

Pontificia Universidad Católica del Perú

Fullan, M. (2002). El significado del cambio educativo: un cuarto del siglo del aprendizaje. Profesorado, revista de curriculum y formación del profesorado, 6 (1-2), 1-14. Recuperado de: https://www.ugr.es/ recfpro/rev61ART1.pdf

Hord, S. (1997). Professional learning communities: communities of continuos Inquiry and improvement. Recuperado de: https://files.eric.ed.gov/fulltex/ED410659.pdf.

Hord, S. (2009). Professional learning communities. Educators work together toward a shared purpose improved student learning.

Recuperado de:hhttp://www.earlychilhoodleadership.com.au/dloads/rsc/professional $\% 20$ communities\%20hord2009.pdf.

Jones, L., Stall, G. \& Yarbrough, D. (2013) The Importance of Professional Learning Communities for School Improvement. College of Education, Nicholls State University, Thibodaux, USA. Creative Education. 4(5), 357-361. Recuperado de https://www.scirp.org/journal/ce

Kruse, S \& Johnson, B. (2017). Tempering the normative demands of professional learning communities with the organizational realities of life in schools: Exploring the cognitive dilemmas faced by educational leaders. Educational Management Administration \& Leadership. 45(4) 588-604 DOI: 10.1177/1741143216636111journals.sagepub.

com/home/ema

Krichesky, G. y Murillo, F. (2011). Las comunidades Profesionales de aprendizaje. Una estrategia de mejora para una nueva concepción de escuela. REICE. Revista Iberoamericana sobre Calidad, Eficacia y Cambio en Educación, 9 (1), 65-83. Recuperado de: http://www.redalyc.org/html/551/55118790005/

La Torre, A, Del Rincón, D, Arnal, J. (1996). Bases metodológicas de la investigación educativa. GR92 Barcelona

Leithwood, K. y Louis, K. S. (1998). Organizational learning in schools. An Introduction. Lisse Netherlands: Swets \& Zeitlinger Publishers.

Leithwood, k; Seashore Louis, K; Anderson, S. \& Wahlstrom, K (2004). Review of research How leadership influences student learning. CAREI. University of Minesota. The Wallace Foundation.

Recuperado de: https://conservancy.umn.edu/bitstream/handle/11299/2035/CAREI

Experiencias de directivos de escuela: El rol del director: cómo lograr los aprendizajes de los estudiantes - Guerra, Gladys.

DOI: https://doi.org/10.18800/cige2020.010 
Congreso Internacional de Gestión Educativa, 06 y 07 de noviembre de 2020.

Pontificia Universidad Católica del Perú

Michele, Y. \& Santos, I. (2016). Problem Finding in Professional Learning Communities: A Learning Study Approach. Scandinavian Journal of Educational Research, 60 (2). 127 146. doi:org/10.1080/00313831.2014 996596

Molina, E. (2005). Creación y desarrollo de comunidades de aprendizaje hacia la mejora educativa. Revista de Educación. Núm. (337), 235-250. Recuperado de:

http://cmapspublic2.ihmc.us/rid=1279042516328_433996408_20112/comunidades\%20d e\%20aprendizaje.pdf

Murillo, F. \& Krichesky, G. (2012). El proceso del cambio escolar. Una guía para impulsar y sostener la mejora de las escuelas. REICE. Revista Iberoamericana sobre Calidad, Eficacia y Cambio en Educación, 10 (1), 26-43. Recuperado de: http://revistas.uam.es/index.php/reice/article/view/3072/3284

Murillo, F. y krichesky, G. (2014). Mejora de la escuela: Medio siglo de lecciones aprendidas. School Impromvement: A half-century of learned lessons. REICE. Revista Iberoamericana sobre Calidad, Eficacia y Cambio en Educación. 13(1) 69-102. Recuperado de: https:// www.rinace.net.reice

Murillo, F. \& krichesky, G. (2015). Mejora de la Escuela: Medio siglo de lecciones aprendidas REICE. Revista Iberoamericana sobre Calidad, Eficacia y Cambio en Educación, 13 (1) (enero-marzo, 2015), 69-102 Red Iberoamericana de Investigación Sobre Cambio y Eficacia Escolar Madrid, España. Recuperado de: http://www.redalyc.org/html/551/55133776005/

Olivier, D., Hipp, K., y Huffman, J. B. (2003). Professional learning community assessment. In J. B. Huffman, \& K. K. Hipp (Eds.), Reculturing schools as professional learning communities, 70-74. Lanham, MD: The Scarecrow Press. Recuperado de: https:// www.scirp.org

Oh Song, K. y Choi, J. (2017). Structural analysis of factors that influence professional learning communities in Korean elementary schools. International Electronic Journal of Elementary Education, 10 (1), 1-9. DOI: 10.26822/iejee.2017131882

Pérez- Ruiz, A. (2014). Enfoques de la gestión escolar: una aproximación desde el contexto latinoamericano. Educación y Educadores, 17(2), 357-369. Doi.10.5294/edu.2014.17.

Rodríguez, J. y Trujillo, J. (2007). ¿Las universidades son organizaciones que aprenden adecuadamente? Universia Business Review, 15, 100-119. Recuperado de: https://www.redalyc.org/df/433/43301507.pdf

Senge, P. (1992). La Quinta Disciplina: Cómo impulsar el aprendizaje en la organización inteligente. Buenos Aires, Argentina: Granica

Experiencias de directivos de escuela: El rol del director: cómo lograr los aprendizajes de los estudiantes - Guerra, Gladys.

DOI: https://doi.org/10.18800/cige2020.010 
Congreso Internacional de Gestión Educativa, 06 y 07 de noviembre de 2020.

Pontificia Universidad Católica del Perú

Santos, M. (2001). La escuela que aprende. Madrid, España: Morata.

Stake, R. (1999). Investigación con estudio de casos. Ediciones Morata, SL. Madrid

Stoll, L., Bolam, R., McMahon, A., Wallace, M., \& Thomas, S. (2006). Professional learning communities: A review of the literature. Journal of educational Change, 7(4), 221-258. Recuperado de: https://doi.org/10.1007/s10833-006-0001-8

Voelkel, R. \& Chrispeels, J. (2017). Within School Differences in Professional Learning Community Effectiveness. Implication for Leadership. Journal of School Leadership, (27), 424-453. Recuperado de: https://journals.sagepub.com/doi/abs/10.1177/ 105268461702700305

Experiencias de directivos de escuela: El rol del director: cómo lograr los aprendizajes de los estudiantes - Guerra, Gladys.

DOI: https://doi.org/10.18800/cige2020.010 